\title{
EDITORIAL
}

\section{It's Official: NeuroRx is Now Neurotherapeutics}

With this issue, the journal of the American Society for Experimental NeuroTherapeutics (ASENT) is assuming the new name Neurotherapeutics. Although the former title NeuroRx was intended by the founders of the journal to serve as shorthand for "Neurotherapeutics," this was not well understood by much of our readership. The editors have selected the new name to better convey the focus of the journal and to more closely align it with its sponsoring society. The MEDLINE/PubMed records for articles in prior issues will contain both titles.

The journal name change comes at a propitious time for several reasons. First, the current issue is by far our largest, and for the first time critically focuses on a single class of disorders. Our guest editors Roger Porter and Jacqueline French are well recognized for their expertise in the therapy of epilepsy, and they have recruited an outstanding group of experts to review developing therapeutics in this area. In addition to discussing virtually every new antiepileptic drug in clinical trials, this issue includes several tour de force overviews ranging from molecular neuroscience to clinical research methodologies. Over the next few years, journal issues will be dedicated to such focused topics as channelopathies, movement disorders, Alzheimer's disease, neuroimmu- nology, and neuro-oncology. Each issue is intended to serve as a critical reference work in its field. Second, last January Elsevier took over as publisher and began a campaign to enhance the visibility and readership of the journal. In addition, the accessibility of the journal has increased, as it is now available through ScienceDirect, Elsevier's digital journals archive.

Third, during the past year the advocacy community has become more actively involved in ASENT activities and the society has emphasized its commitment to enhancing educational activities for this important constituency. Neurotherapeutics will serve an important role in this mission. Similarly, the journal will support the commitment of ASENT leadership to promote the training of clinician investigators, which includes a didactic training program to be offered in association with the March 2007 annual meeting in Washington, D.C. Collectively these actions are intended to promote the mission of ASENT to facilitate the development of more effective neurotherapeutic drugs and devices.

Alan I. Faden, M.D. E-mail: fadena@georgetown.edu Editor-in-Chief 